\title{
Calidad sensorial de la carne de cabritos lechales criados en sistemas de producción basados en pastoreo
}

Francisco De-la-Vega Galán ${ }^{a^{*}}$

José Luis Guzmán Guerrero b

Manuel Delgado Pertíñez ${ }^{\text {a }}$

Luis Ángel Zarazaga Garcés ${ }^{b}$

Pilar Ruiz Pérez-Cacho ${ }^{\mathrm{c}}$

Hortensia Galán-Soldevilla ${ }^{\mathrm{c}}$

a Universidad de Sevilla, Escuela Técnica Superior de Ingeniería Agronómica, Departamento de Ciencias Agroforestales, Sevilla, España.

b Universidad de Huelva "Campus de Excelencia Internacional Agroalimentario, ceiA3", Escuela Técnica Superior de Ingeniería, Departamento de Ciencias Agroforestales. Campus Universitario de la Rábida, Carretera de Huelva-Palos de la Frontera. s/n. 21819, Huelva, España.

${ }^{c}$ Universidad de Córdoba, E.T.S. de Ingenieros Agrónomos y Montes, Departamento de Bromatología y Tecnología de los Alimentos, Córdoba, España.

* Autor de correspondencia: fdelavega@colvet.es

\section{Resumen:}

En España, hay un interés creciente por la conservación de las razas caprinas autóctonas con formas de producción basadas en el pastoreo y por la posibilidad de transformación en producciones ecológicas. El objetivo ha sido evaluar las características sensoriales de la carne de cabrito lechal de dos razas autóctonas, criados en sistemas de producción convencional y ecológica, basados en el pastoreo. Se utilizaron 21 cabritos lechales de los cuales 12 fueron criados en un sistema ecológico (6 de raza Payoya y 6 de raza Blanca Andaluza) y 9 en un sistema convencional (3 de raza Payoya y 6 de raza Blanca Andaluza). El perfil sensorial de la carne se evaluó mediante un panel analítico. Con 
relación al sistema de explotación, los resultados obtenidos indican que las carnes procedentes de sistemas ecológicos tenían menos intensidad de olor y una textura más blanda, tierna y jugosa que las carnes del sistema convencional. Respecto a la raza, el estudio mostró que las carnes de cabrito de la raza Blanca Andaluza presentaron una intensidad de olor menor y una textura más blanda, tierna y jugosa que las carnes de cabrito de la raza Payoya. Estos resultados preliminares podrían ser favorables para la transformación de las explotaciones convencionales, basadas en pastoreo, a ecológicas.

Palabras clave: Calidad sensorial, Carne, Cabrito, Ecológico.

Recibido: 15/07/2019

Aceptado: $13 / 02 / 2020$

Actualmente hay un interés creciente, por parte de la administración y de los ganaderos españoles, en la conservación de las razas autóctonas, criadas en sistemas extensivos o semiextensivos, basados en el pastoreo. Muchas de estas razas, como es el caso de las razas caprinas Blanca Andaluza y Payoya, están consideradas como razas en peligro de extinción ${ }^{(1)}$, estando la mayor parte de las explotaciones localizadas en zonas de sierra de difícil acceso, en la Comunidad Autónoma de Andalucía ${ }^{(2)}$.

La raza Blanca Andaluza es una raza de aptitud cárnica. En la actualidad, aunque se sigue produciendo el "chivo" (animal criado a pasto con la madre, sacrificado a los 5 meses de edad con 25-30 kg. de peso vivo), principalmente en zonas donde es tradicional el consumo de la carne de estos animales, el tipo comercial más en uso es "cabrito lechal"(2). La raza Payoya es una de las mejores representantes de las razas de producción de leche basada en pastoreo. El principal objetivo de las explotaciones es la producción láctea y de manera secundaria la de carne de "cabrito lechal", sacrificado con un peso de 8 a $9 \mathrm{~kg}$, favorecido por su alto precio en el mercado.

De acuerdo con los requerimientos de la producción ecológica, las explotaciones de estas razas pueden ser fácilmente trasformados en explotaciones ecológicas ${ }^{(3,4)}$. El estudio de las posibilidades de transformación necesita, además del análisis de viabilidad técnica y económica, el estudio de la calidad de los productos. En este sentido, tomando como base las mismas explotaciones que las utilizadas en el presente trabajo, se publicaron dos trabajos sobre el perfil de ácidos grasos ${ }^{(5,6)}$ y dos trabajos sobre la calidad de la carne ${ }^{(7,8)}$, en los que se concluye que no hay diferencias significativas en la gran mayoría de los ácidos grasos presentes en la grasa intramuscular, o en otros depósitos grasos entre los cabritos criados en explotaciones convencionales y ecológicas, así como en la mayoría de los atributos de la carne, con lo que la transformación, desde este punto de vista, podría realizarse fácilmente. Puesto que no se conocen trabajos sobre la calidad sensorial de la carne de cabrito de estas razas autóctonas y teniendo en cuenta los trabajos anteriores, la hipótesis planteada es 
que no habría diferencias importantes en la calidad sensorial de la carne según el sistema convencional y ecológico en ambas razas, lo que facilitaría la transformación de las granjas convencionales al sistema ecológico. Por ello, el objetivo de este estudio ha sido estudiar la calidad sensorial de la carne de los cabritos de las razas Payoya y Blanca Andaluza en sistemas de producción ecológicos y convencionales basados en el pastoreo.

Para este trabajo se utilizaron cabritos lechales pertenecientes a cuatro explotaciones caprinas con sistemas semi-extensivos ${ }^{(5,6)}$, una de cada tipo de producción (convencional y ecológica, con certificación Reglamento según el (CE) nº 834/2007 del Consejo $^{(9)}$ y de cada raza (Payoya y Blanca Andaluza). Las cabras de todas las explotaciones han tenido una alimentación basada en pastos naturales tipo arbustivo Mediterráneo. El área de estudio está dominada por arbustos (60 a $80 \%$ de cobertura y alrededor de 0.6 a $1.8 \mathrm{~m}$ de altura) y árboles (principalmente Mirtus communis, Pistacia lentiscus, Quercus ilex, Cistus salvifolius and Arbutus unedo). También hay pastos formados por especies de gramíneas (Lolium spp, Phalaris acuatica, Hainardia cylindrica, Hordeum bulbosum), leguminosas (Trifolium subterraneum, T. pallidum, T. aquamosum, T. squarrosum, T. istmocarpum, Scorpiurus muricatus, S. vermiculatus, son las más comunes) y otras familias de dicotiledóneas (Cichorium spp., Carlina racemosa, Cynara humilis, Echium plantagineum, Galactites tomentosa, Scolymus spp., son las más comunes). Independientemente de la disponibilidad de pastos, los animales de ambas razas pastan durante todos los días del año, aunque las cabras se mantienen en los corrales durante la tarde-noche para el amamantamiento de los cabritos (en ambas razas). Los pastos se complementan en todos los casos con el suministro de concentrados. En el presente estudio se utilizaron suplementos con alimentos concentrados a razón de $1 \mathrm{~kg}$ y $0.5 \mathrm{~kg}$ por cabeza y día en las explotaciones convencionales y ecológicas, respectivamente, de raza Payoya; así como 0.6 kg y 0.35 $\mathrm{kg}$ en las explotaciones convencionales y ecológicas, respectivamente, de raza Blanca Andaluza (Cuadro 1).

Cuadro 1: Ingredientes y composición química de los concentrados suplementados en los sistemas de producción convencional y ecológico

\begin{tabular}{lllll}
\hline $\begin{array}{l}\text { Ingredientes (\% de materia } \\
\text { fresca) }\end{array}$ & \multicolumn{2}{l}{ Payoya } & \multicolumn{2}{l}{ Blanca Andaluza } \\
\cline { 2 - 5 } & Convencional & Ecológico & Convencional Ecológico \\
\hline Granos de cebada & 10.0 & 74.0 & - & 74.0 \\
Habas & - & - & 60.0 & - \\
Pulpa de remolacha & 9.5 & - & - & - \\
Algarroba & - & 4.0 & - & 4.0 \\
Grasa by-pass & 1.5 & - & - & - \\
Gluten feed & 12.0 & - & - & - \\
Guisantes & - & 5.0 & 40.0 & 5.0 \\
Granos de maíz & 26.0 & - & - & - \\
Harina de soja & 18.2 & - & - & - \\
Melaza caña de azúcar & 2.0 & - & - & -
\end{tabular}




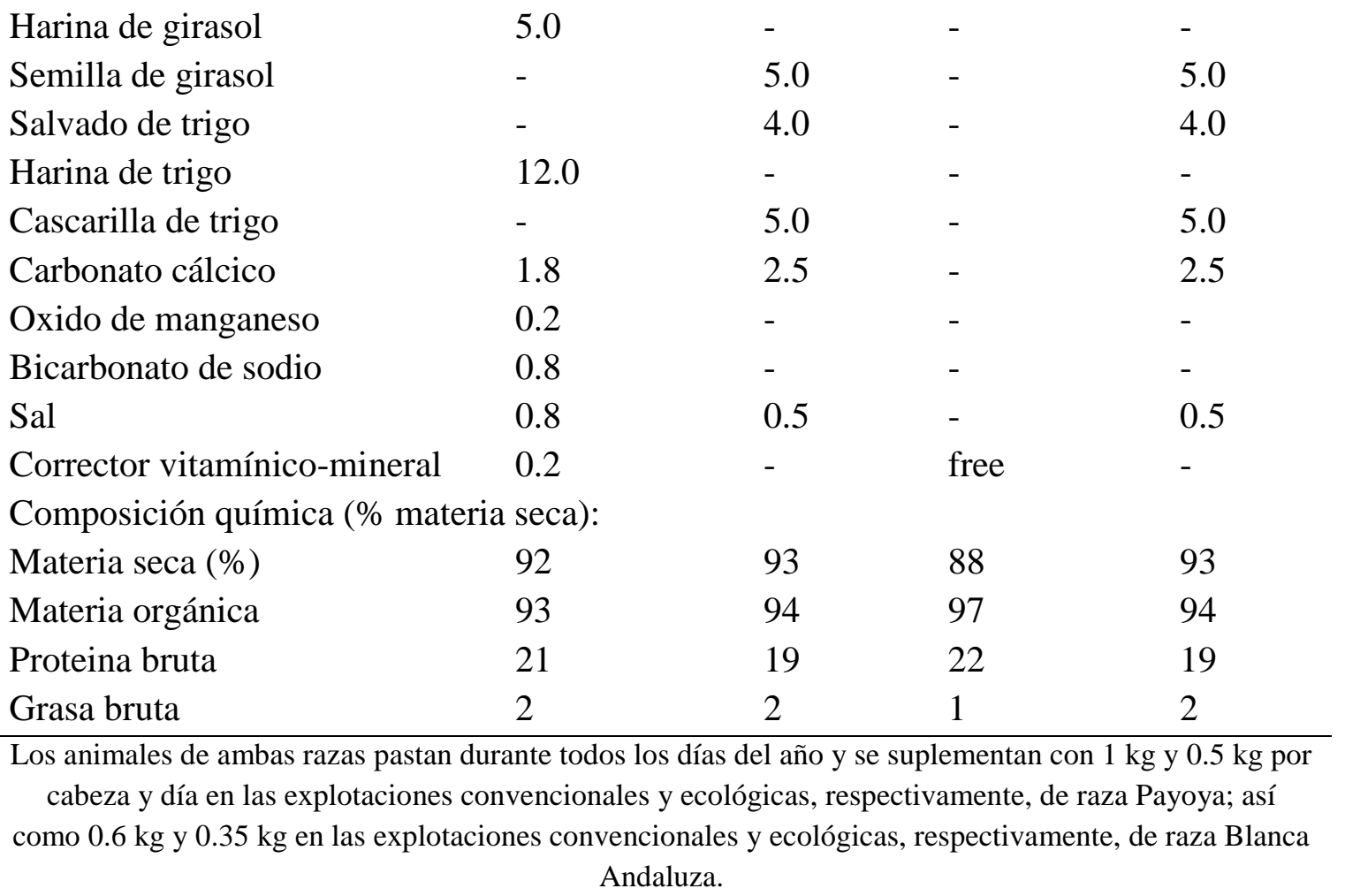

Se utilizaron 21 cabritos lechales, nacidos de partos dobles: 12 cabritos criados en un sistema ecológico (6 de raza Payoya y 6 de raza Blanca Andaluza) y 9 cabritos criados en un sistema convencional ( 3 de raza Payoya y 6 de raza Blanca Andaluza). Las cabras y los cabritos de cada una de las granjas fueron seleccionados al azar dentro de la misma estación. Los cabritos, durante todo el periodo de lactación, tuvieron acceso a las madres, pero no a otros alimentos.

Todos los cabritos se sacrificaron con un peso vivo de $8.12 \pm 0.49 \mathrm{~kg}$ (Payoya) y $7.52 \pm$ $0.64 \mathrm{~kg}$ (Blanca Andaluza) en un matadero oficial de Huelva (España), después de $16.00 \pm 0.75 \mathrm{~h}$ (Payoya) y $19.81 \pm 2.49 \mathrm{~h}$ (Blanca Andaluza) de ayuno con libre acceso al agua. Después del sacrificio, las canales se refrigeraron a $4{ }^{\circ} \mathrm{C}$ durante un período de maduración de $24 \mathrm{~h}$. A continuación, se obtuvo la media canal izquierda que se transportó refrigerada a las instalaciones de la Universidad de Huelva. Allí se procedió al despiece y obtención de las piernas ${ }^{(10)}$, que se envasaron al vacío y se congelaron a $-20{ }^{\circ} \mathrm{C}$ hasta su posterior análisis. Los pesos medios de las piernas fueron de $0.65 \pm 0.03$ $\mathrm{kg}$ en los cabritos de raza Payoya y $0.56 \pm 0.07 \mathrm{~kg}$ en los de raza Blanca Andaluza.

Previamente al análisis sensorial, las piernas se descongelaron dentro de la bolsa de vacío, por inmersión en agua corriente a una temperatura de 17 a $19{ }^{\circ} \mathrm{C}$. Las piernas enteras se cocinaron en un horno eléctrico, hasta llegar a una temperatura interna de 65 a $70{ }^{\circ} \mathrm{C}$, controlada con un termopar (JENWAY 2000). Una vez cocinada la carne, se extrajo el músculo Semimembranosus y se cortó en submuestras de 2 x $2 \mathrm{~cm}$, siendo posteriormente envueltas individualmente en papel de aluminio, previamente codificado con un número aleatorio de tres cifras. Las muestras se sirvieron en las cabinas de cata, 
donde se mantuvieron calientes en un horno eléctrico precalentado a $60{ }^{\circ} \mathrm{C}$ hasta el momento del análisis. Posteriormente se sirvieron aleatoriamente de una en una a un panel entrenado de siete catadores, seleccionados y entrenados según las normas internacionales ISO $8586^{(11)}$, y pertenecientes al panel analítico del Laboratorio Sensorial del Departamento de Bromatología y Tecnología de los Alimentos de la Universidad de Córdoba. Las muestras de las 21 piernas se analizaron en seis sesiones de no más de 1 h de duración, evaluándose un máximo de cuatro muestras por sesión. La metodología seguida es una adaptación de la propuesta por Guerrero ${ }^{(12)}$ para el análisis sensorial de carnes de pequeños rumiantes. Todos los análisis se realizaron en horario de mañana (1200 a 1300 h) en la sala de cata de la Escuela de Hostelería de Córdoba. Entre muestras, los jueces utilizaron agua mineral para limpiarse las papilas. Se analizaron seis atributos sensoriales: 1 de apariencia (intensidad de color), 1 de olor (intensidad global de olor), 3 de textura (dureza, facilidad de masticación y jugosidad) y 1 de aroma (intensidad global de aroma) en una escala lineal no estructurada de $10 \mathrm{~cm}$ de longitud anclada a $1 \mathrm{~cm}$ de sus extremos. Además, se evaluaron cualitativamente las notas de olor (ortonasal) y aroma (retronasal) y los sabores básicos.

Los atributos sensoriales cuantitativos se analizaron mediante un análisis de varianza de dos vías (sistema de explotación x raza), usando el Modelo Lineal General (GLM) del paquete estadístico IBM SPSS para Windows (versión 22.0; IBM Corp., Armonk Nueva York, USA). Además, se realizó un análisis de varianza (catador) para cada atributo sensorial con el objetivo de evaluar si el panel trabajaba como un grupo. Con todos los parámetros analizados se realizó un análisis factorial utilizando el método de los componentes principales (CP) y seleccionando aquellos factores con un autovalor asociado mayor a 1 .

Los resultados indicaron que para todos los atributos sensoriales cuantificados el panel trabajó como grupo $(P>0.05)$. En el Cuadro 2 se presentan los valores medios, las desviaciones estándar y el análisis de varianza (sistema de explotación x raza) para cada atributo sensorial. El análisis de varianza muestra que existen diferencias significativas entre formas de producción $(P<0.05)$ para todos los atributos sensoriales analizados excepto para la intensidad de color y la intensidad global de aroma; diferencias significativas entre razas para los atributos intensidad global de olor $(P<0.001)$, dureza $(P<0.01)$ y jugosidad $(P<0.01)$ e interacciones (sistema explotación x raza) para los atributos intensidad de color $(P<0.001)$ y dureza $(P<0.05)$. 
Cuadro 2: Medidas descriptivas (valores medios y error estándar de la media) y análisis de la varianza (sistema de explotación x raza) de los atributos sensorial de las carnes de cabrito analizadas

\begin{tabular}{|c|c|c|c|c|c|c|c|}
\hline \multirow[b]{2}{*}{ Atributos } & \multicolumn{2}{|c|}{$\begin{array}{l}\text { Sistema de explotación } \\
\text { (SE) }\end{array}$} & \multicolumn{2}{|c|}{$\operatorname{Raza}(\mathbf{R})$} & \multicolumn{3}{|c|}{ Significación } \\
\hline & $\begin{array}{l}\text { Ecológico } \\
(n=12)\end{array}$ & $\begin{array}{l}\text { Convencional } \\
(n=9)\end{array}$ & $\begin{array}{l}\text { Blanca } \\
(n=12)\end{array}$ & $\begin{array}{l}\text { Payoya } \\
(n=9)\end{array}$ & SE & $\mathbf{R}$ & $\begin{array}{l}\text { SE } \times \\
\mathbf{R}\end{array}$ \\
\hline $\begin{array}{l}\text { Intensidad de } \\
\text { color }\end{array}$ & $4.9 \pm 0.19$ & $5.2 \pm 0.16$ & $5.1 \pm 0.16$ & $5.0 \pm 0.19$ & ns & ns & $* * *$ \\
\hline $\begin{array}{l}\text { Intensidad global } \\
\text { olor }\end{array}$ & $5.8 \pm 0.07$ & $6.2 \pm 0.08$ & $5.8 \pm 0.08$ & $6.1 \pm 0.05$ & $* * *$ & $* * *$ & ns \\
\hline Dureza & $4.3 \pm 0.11$ & $5.0 \pm 0.17$ & $4.5 \pm 0.13$ & $4.7 \pm 0.17$ & $* * *$ & $* *$ & $*$ \\
\hline $\begin{array}{l}\text { Facilidad de } \\
\text { masticación }\end{array}$ & $5.0 \pm 0.15$ & $4.5 \pm 0.19$ & $4.9 \pm 0.16$ & $4.5 \pm 0.17$ & $* * *$ & ns & ns \\
\hline Jugosidad & $4.1 \pm 0.12$ & $3.8 \pm 0.16$ & $4.2 \pm 0.15$ & $3.7 \pm 0.07$ & $*$ & $* *$ & ns \\
\hline $\begin{array}{l}\text { Intensidad global } \\
\text { aroma }\end{array}$ & $5.4 \pm 0.07$ & $5.8 \pm 0.10$ & $5.4 \pm 0.07$ & $5.8 \pm 0.09$ & ns & ns & ns \\
\hline
\end{tabular}

$* P<0.05 ; * * P<0.01 ; * * * P<0,001 ;$ ns $=$ no significativo.

Así, en el presente estudio se observó que las carnes procedentes de sistemas ecológicos tenían menos intensidad de olor y una textura más blanda, tierna y jugosa que las carnes del sistema convencional. Utilizando los mismos animales que en el presente estudio, los parámetros de capacidad de retención de agua (CRA) y textura o resistencia al corte de la carne no presentaron diferencias significativas entre sistemas en ambas razas $^{(7,8)}$ excepto en la raza Payoya, en la que los cabritos de sistema ecológico presentaron una mayor CRA, lo que contribuiría a explicar, al menos parcialmente, los resultados positivos obtenidos en los cabritos ecológicos de esta raza. Aunque no hay referencias bibliográficas de trabajos similares que establezcan diferencias sensoriales entre los cabritos lechales pertenecientes a ambos sistemas, sí hay algunos trabajos que han estudiado las diferencias observadas entre diferentes regímenes de alimentación. Así, en el trabajo de Costa et $a l^{(13)}$ sobre el perfil sensorial de carne de cabritos de la raza Blanca Serrana Andaluza con pesos de $19 \mathrm{~kg}$, sólo encontraron diferencias para la intensidad global de aroma en los cabritos criados en sistemas intensivos (6.2) en comparación a los criados en sistemas extensivos (5.2), explicando estas diferencias en función de un mayor contenido en grasa en los cabritos en intensivo. Otros autores ${ }^{(14)}$ también han encontrado diferencias sensoriales en la carne de cabritos lechales alimentados con leche natural o con un sustituto de leche, observando un mayor olor y sabor en los cabritos alimentados con este último, a pesar de no encontrar diferencias en el porcentaje de grasa intramuscular entre las dos dietas. Estos autores indicaron que las diferencias podrían estar relacionadas con las variaciones en el grado de insaturación de esa grasa intramuscular como consecuencia de las diferencias en la alimentación. 
En el presente trabajo y de forma general, la alimentación recibida por las madres ha sido similar, por lo que las variaciones en el perfil sensorial de las carnes entre explotaciones se podrían atribuir a los aportes nutritivos procedentes del pastoreo y a los concentrados suplementados ${ }^{(5,6)}$. Además, las carnes de cabrito estudiadas ${ }^{(5,6)}$ no presentaron diferencias significativas en el contenido de grasa intramuscular, no obstante los porcentajes de los ácidos grasos C17:0, C17:1, C20:1, C20:4 n-6, C22:2 y algunos ácidos grasos n-3 (el ácido omega-3 docosahexaenoico C22:5 -DPA- y el ácido C22:6 -DHA-), fueron más altos en la grasa intramuscular de los cabritos ecológicos de raza Blanca Andaluza ${ }^{(5)}$ y los porcentajes de C14:0, C18:1 trans-11- (VA) y de los ácidos grasos n-3 C20:5 (EPA), DHA y DPA fueron también más altos en la grasa intramuscular de los cabritos ecológicos de raza Payoya ${ }^{(6)}$, lo que podría explicar las diferencias en estos atributos sensoriales. En este sentido, en un estudio reciente sobre la alimentación con sustituto de leche (16\% de materia seca) de cabritos ${ }^{(15)}$, la evaluación de la calidad sensorial de la carne ha mostrado que la adición de una alta dosis de DHA (1.8\%) da lugar a carnes con olor y sabor desagradables y puntuaciones bajas de aceptación general, en comparación a dosis bajas $(0.9 \%)$, lo que podría indicar que al ser animales muy jóvenes este ácido graso seguramente se depositó en la grasa intramuscular en cantidades altas y eso daría lugar a una valoración sensorial inferior. En nuestro trabajo no se ha determinado la ingestión de DHA en la dieta de los cabritos, pero, aunque los animales ecológicos han presentado significativamente un mayor porcentaje de DHA en la carne $(0.13$ a $0.19 \%$ en los cabritos ecológicos y 0.09 a 0.10 $\%$ en los convencionales), sus características sensoriales no han sido mayores que la de los cabritos convencionales. La pequeña diferencia en este ácido n-3 entre cabritos, junto al efecto y relaciones con otros ácidos grasos podrían explicar los resultados sensoriales encontrados en el presente estudio.

Respecto a la raza, el estudio indicó que las carnes de cabrito de la raza Blanca Andaluza presentaron una intensidad de olor menor y una textura más blanda, tierna y jugosa que las carnes de cabrito de la raza Payoya. Los resultados podrían explicarse parcialmente por la mayor textura de la carne de los cabritos de raza Payoya (7.26 $\left.\mathrm{kg} / \mathrm{cm}^{2}\right)$ que la de los cabritos de la raza Blanca Andaluza $\left(5.59 \mathrm{~kg} / \mathrm{cm}^{2}\right)(P<0,001$, datos no publicados). Diversos autores también han encontrado algunas diferencias sensoriales entre razas o diferentes genotipos ${ }^{(16,17)}$ pero apenas hay publicaciones que estudien la calidad sensorial de las dos razas autóctonas estudiadas en el presente trabajo y ninguno que las compare. Como ya indicaba Sañudo ${ }^{(18)}$, la raza es un factor que puede hacer variar la calidad del producto y que en muchos casos justifica, por si sola, la existencia de marcas de calidad.

En Cuadro 3 se presentan los resultados del análisis cualitativo para cada raza según la forma de producción. En relación con la raza Blanca andaluza, los resultados muestran diferencias para los descriptores del olor y aroma y los sabores básicos para las muestras estudiadas dependiendo de la forma de producción: las carnes del sistema ecológico fueron descritas con notas olfativas a carne de cocido y sabor metálico mientras que las carnes del sistema convencional además de la nota olfativa carne de cocido, tenían 
olor/aroma a hígado y cabrito y un sabor ácido. En cambio, en la raza Payoya no se observan claras diferencias entre ambas formas de producción para los descriptores de olor y aroma, pero sí para los sabores básicos. En este sentido, aunque las carnes de ambas formas de producción son metálicas, la ecológica es además sabrosa.

Cuadro 3: Frecuencias (\%) de los descriptores del análisis cualitativo (olor/aroma y sabores básicos) de la carne de cabrito lechal, para cada raza y forma de producción

\begin{tabular}{lcccc}
\hline Descriptor & \multicolumn{3}{c}{ Blanca Andaluza } & Payoya \\
\cline { 2 - 5 } & $\begin{array}{c}\text { Ecológico } \\
(\mathbf{n = 6})\end{array}$ & $\begin{array}{c}\text { Convencional } \\
(\mathbf{n = 6})\end{array}$ & $\begin{array}{c}\text { Ecológico } \\
(\mathbf{n = 6})\end{array}$ & $\begin{array}{c}\text { Convencional } \\
(\mathbf{n}=\mathbf{3})\end{array}$ \\
\hline Olor y aroma: & 100 & 67 & 83 & 33 \\
Carne de cocido & 17 & 100 & 50 & 67 \\
Hígado & 0 & 50 & 0 & 0 \\
Cabrito & 0 & 17 & 0 & 0 \\
Orina & & & & \\
Sabores básicos: & 17 & 17 & 50 & 0 \\
Sabrosa & 67 & 0 & 50 & 67 \\
Metálico & 0 & 50 & 17 & 0 \\
Acida & & & \\
\hline
\end{tabular}

Para intentar agrupar las muestras según la forma de producción y la raza de procedencia se realizó un análisis de componentes principales. Los dos primeros $\mathrm{CP}$ explican casi el $66 \%$ del total de la varianza de los atributos de calidad sensorial (37.86 y $27.88 \%$, para el primer y segundo componente respectivamente). El $\mathrm{CP}_{1}$ estaría formado principalmente por las siguientes medidas de la textura de la carne: por un lado, la facilidad de masticación y la jugosidad, situados a la derecha del gráfico, y por otro, la dureza situada a la izquierda del gráfico (Figura 1a). El $\mathrm{CP}_{2}$ se caracteriza por la intensidad del olor y del aroma, ambos atributos de calidad situados en la parte superior del gráfico. La Figura 1b muestra la proyección de los cabritos, para las dos formas de producción, en el plano definido por los dos CP. Aunque las muestras de carne han presentado gran variación, el sistema ecológico está preferentemente localizado en la parte derecha (mayor jugosidad y facilidad de masticación y menor dureza) e inferior (menos intensidad de olor y aroma) de la figura, mientras que el sistema convencional se localiza preferentemente en la parte izquierda y superior. La Figura 1c muestra la proyección de los cabritos para las razas, en el plano definido por los dos CP. A pesar de la variabilidad de los datos al igual que para el sistema, se pueden observar dos grupos, uno en la parte inferior del gráfico que se corresponde con la raza Blanca Andaluza (menos intensidad de olor y aroma) y otro a la izquierda del gráfico correspondiente a la raza Payoya (menos jugosidad y facilidad de masticación). 
Figura 1: Resultados del análisis factorial de componentes principales (CP)

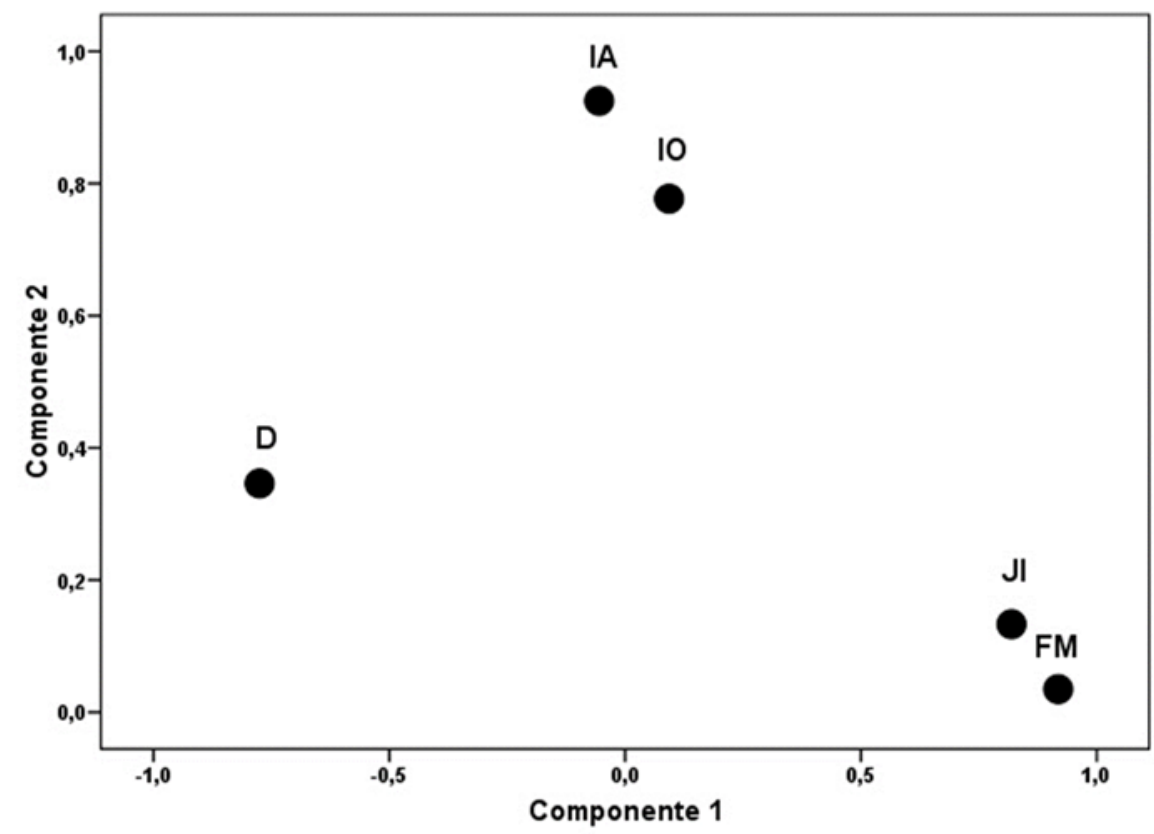

D: Dureza. IA: Intensidad de Aroma. I0: Intesidad de Olor. Jl: Jugosidad Inicial. FM: Facilidad de Masticación. D: Terderness. IA: Aroma Intensity. 10: Odor Intensity. Jl: Initial Juiciness. FM: Mastication.

a) Proyección de los atributos de calidad sensorial de la carne de cabrito en el plano definido por los dos componentes principales.

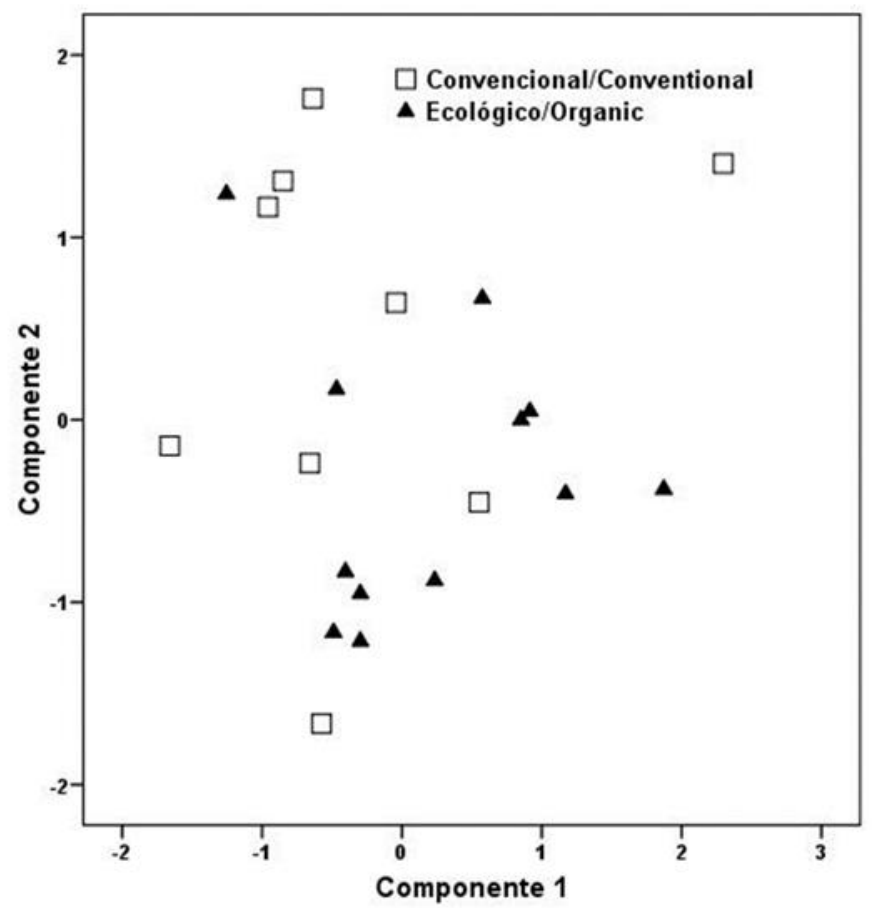

b) Proyección de los cabritos, para los dos sistemas de producción estudiados, en el plano definido por los dos componentes principales 




c) Proyección de los cabritos, para las dos razas estudiadas, en el plano definido por los dos componentes principales

En conclusión, los cabritos procedentes del sistema ecológico y los procedentes de la raza Blanca andaluza, en comparación a los del sistema convencional y de la raza Payoya, presentaron en general carnes con mejores atributos sensoriales (más tiernas, más jugosas, con mayor facilidad a la masticación) y menos intensidad de olor. En la raza Blanca andaluza existen claras diferencias entre sistemas para los descriptores del olor y aroma y sabores básicos, en cambio, en la raza Payoya sólo se observan claras diferencias entre sistema para los sabores básicos. Aunque la muestra presenta una gran variabilidad, por lo que son necesarios nuevos estudios con mayor número de animales, estos resultados preliminares serían favorables para promocionar la transformación de las explotaciones convencionales en ecológicas basadas en sistemas de pastoreo.

\section{Agradecimientos}

Los autores agradecen al Instituto Andaluz de Investigación y Formación Agraria, Pesquera, Alimentaria y de la Producción Ecológica de la Consejería de Agricultura y Pesca de la Junta de Andalucía por la financiación del proyecto ( $\left.\mathrm{N}^{\circ} 75,92162 / 1\right)$, a los ganaderos Francisca Delgado Méndez, Domingo Ginés Domínguez, Benjamín Bombas González, Manuel Sánchez Sánchez y Daniela Hinojo Antille por contribuir con la aportación de sus animales y al panel analítico del Laboratorio Sensorial del Departamento de Bromatología y Tecnología de los Alimentos de la Universidad de Córdoba. 


\section{Literatura citada:}

1. Real Decreto 2129/2008, de 26 de diciembre, por el que se establece el Programa nacional de conservación, mejora y fomento de las razas ganaderas. B.O.E. $n^{\circ} 23$, martes 27 de enero de 2009: 9211-9242.

2. FEAGAS. Federación Española de Asociaciones de Ganado Selecto 2014; 38.

3. Mena Y, Ligero M, Ruiz FA. Organic and conventional dairy goat production systems in Andalusian mountainous areas. Option Méditerranéennes A 2009;91:253-256.

4. Mena Y, Nahed J, Ruiz FA. Proximity to the organic model of dairy goat systems in the Andalusian mountains (Spain). Trop Subtrop Agroecosyst 2009;11:69-73.

5. De-La-Vega F, Guzmán JL, Delgado-Pertíñez M, Zarazaga LA, Argüello A. Fatty acid composition of muscle and adipose tissues of organic and conventional Blanca Andaluza suckling kids. Spanish J Agric Res 2013;1(3):770-779.

6. De-La-Vega F, Guzmán JL, Delgado-Pertíñez M, Zarazaga LA, Argüello A. Fatty acid composition of muscle and internal fat depots of organic and conventional Payoya goat kids. Spanish J Agric Res 2013;11(3):759-769.

7. Guzmán JL, De-la-Vega F, Zarazaga LA, Argüello A, Delgado-Pertíñez M. Carcass and meat quality of Blanca Andaluza kids fed exclusively with milk from their dams under organic and conventional grazing-based management systems. Italian J Anim Sci 2019;18 (1):1186-1191.

8. Guzmán JL, De-La-Vega F, Zarazaga LA, Argüello A, Delgado-Pertíñez M. Carcass characteristics and meat quality of Payoya breed conventionally and organically reared dairy goat suckling kids. Ann Anim Sci 2019;9(4):1149-1159.

9. Reglamento (CE) $n^{\circ} 834 / 2007$ del Consejo, de 28 de junio de 2007, sobre producción y etiquetado de los productos ecológicos y por el que se deroga el Reglamento (CEE) no 2092/91. Diario Oficial de la Unión Europea L 189: 1-23.

10. Colomer-Rocher F, Morand-Fehr P, Kirton H. Standard methods and procedures for goat carcass evaluation, jointing and tissue separation. Livest Prod Sci 1987;17: 149-159.

11. ISO 8586. International Organization for Standardization Publications. Sensory analysis - Methodology_. General guidance for the selection, training and monitoring of selected assessors and expert sensory assessors. 2012.

12. Guerrero L. Determinación sensorial de la calidad de la carne. En: Cañeque V, Sañudo C editores. Metodología para el estudio de la calidad de la canal y de la carne en rumiantes, Madrid, Instituto Nacional de Investigación y Tecnología Agraria y Alimentaria 2000;205-220. 
13. Germano Costa R, Galán H, Camacho Vallejo ME, Vallecillo A, Delgado Vermejo JV, Argüello Henríquez A. Perfil sensorial de la carne de cabritos de la raza Blanca Serrana Andaluza. Arch Zoot 2008;57:67-70.

14. Bañon S, Vila R, Price A, Ferrandini E, Garrido MD. Effects of goat milk or milk replace diet on milk quality and fat composition of suckling goat kids. Meat Sci 2006;72:216-221.

15. Moreno-Indias I, Sánchez-Macías D, Martínez de-la-Puente J, Morales de la Nuez A, Hernández-Castellano LE, Castro N, Argüello A. The effect of diet and DHA addition on the sensory quality of goat kid meat. Meat Sci 2012;90:393-397.

16. Lemes JS, Monge P, Campo MM, Guerra V, Sañudo, C. Estudio comparativo de la calidad de productos caprinos locales frente a sus posibles competidores. Congreso de la SEOC 2011;154-157.

17. Ngambu S, Muchenje V, Chimonyo M, Marume U. Correlations among sensory characteristics and relationships between aroma scores, flavour scores, off-flavour scores and off-flavour descriptors of chevon from four goat genotypes. African $\mathbf{J}$ Biotech 2011;10(34):6575-6580.

18. Sañudo C. Calidad de la canal y de la carne ovina y caprina y los gustos de los consumidores. Rev Brasileña Zoot 2008;37:143-160. 